http://jmscr.igmpublication.org/home/ ISSN (e)-2347-176x ISSN (p) 2455-0450

crossref DOI: https://dx.doi.org/10.18535/jmscr/v8i12.41

\title{
Prevalence of Hypothyroidism in Patients with Chronic Kidney Disease in a Tertiary Hospital in South India
}

\author{
Authors \\ Dr Kaveri Kannan ${ }^{1}$, Dr Preethi Shahila ${ }^{2}$, Dr K.R. Jegan ${ }^{3}$, Dr Parvathy. $\mathbf{S}^{4}$ \\ ${ }^{1}$ Associate Professor, Department of General Medicine, Kanyakumari Government Medical College, \\ Asaripallam \\ ${ }^{2}$ Assistant Professor, Department of General Medicine, Kanyakumari Government Medical College, \\ Asaripallam \\ ${ }^{3,4}$ Post Graduate, Department of General Medicine, Kanyakumari Government Medical College, \\ Asaripallam
}

\section{Abstract}

Background: Thyroid hormones have significant impact on kidney disease so it is important to consider the physiological association of thyroid dysfunction in relation to chronic kidney disease (CKD). CKD has been known to affect the pituitary-thyroid axis and the peripheral metabolism of thyroid hormones. Low T3 levels are the most common laboratory finding followed by subclinical hypothyroidism in CKD patients.

Aim: To prove the prevalence of hypothyroidism in CKD patients and its relation between cardiovascular disease and mortality in a tertiary care hospital, in South India.

Materials \& Methods: A cross-sectional study was performed among 100 CKD patients of a tertiary care hospital in South India from September 2018 to November 2018 to determine the prevalence of hypothyroidism in the both dialysis dependent and non-dialysis dependent CKD population.

Observation \& Conclusion: From this study an increased prevalence of hypothyroidism (clinical and subclinical) was observed in patients with reduced glomerular filtration rate and also an increased risk of cardiovascular events in these patients. Patients in the overt hypothyroid group had increased risk of cardiovascular events compared to euthyroid groups

Keywords: Hypothyroidism, CKD, Cardiovascular events.

\section{Introduction}

Thyroid hormones play a very important role regulating metabolism, development, protein synthesis, and influencing other hormone functions. These hormones can also have significant impact on kidney disease so it is important to consider the physiological association of thyroid dysfunction in relation to chronic kidney disease (CKD).
1] Primary hypothyroidism, characterised by elevated serum thyroid stimulating hormone (TSH) level in conjunction with a low or normal thyroxine (T4) level (clinical and subclincal hypothyroidism) is found to be more prevalent in patients with advanced kidney dysfunction compared to those with normal function.

One of the most important conditions that has been less studied is thyroid hormone levels and how they affect the progression of CKD. 
Disorders in renal function have been seen to coexist with specific levels of thyroid hormone. This study is done to simplify the importance of interactions between thyroid function and kidney disease. This information is essential as it shows a link between two separate conditions.

\section{Materials \& Methods}

Study Design: Cross-sectional study

Study Period: September 2018 to November 2018

Study Centre: Department of General Medicine, Kanyakumari Government Medical College, Asaripallam, Tamil Nadu, India

\section{Method}

\section{Inclusion Criteria}

Any adult ( $>18$ years) diagnosed as a CKD patient on the basis of USG KUB, urine routine examination and elevated RFT parameters.

\section{Exclusion Criteria}

- Patients < 18 years of age

- AKI patients

- Patients who are on drugs like amiodarone, thyroxine / s/p thyroidectomy/ ca thyroid.

- Patients who were known case of cardiac diseases.

\section{Observation \& Results}

Among 100 CKD patients, 30 were women and 70 men. Out of 70 men, 40 had systemic hypertension and 30 were normotensive and among women 20 were hypertensive and 10 normotensive. Out of 100 CKD cases $60 \%$ were hypertensive and $40 \%$ normotensive. Out of total cases $60 \%$ were diabetic and $40 \%$ were non diabetic. Out of total 70 men, 55 were diabetic and 15 were non diabetic. Among total 100 cases, 40 were normothyroid, 40 had biochemical subclinical hypothyroidism (elevated TSH and normal T3, T4 levels) and 20 had overt hypothyroidism (elevated TSH and decreased T3 and T4). Out of our 30 females in the study, 20 were found to be hypothyroid. So realtively there is increased prevalence of hypothyroidism among females. Among 20 females 5 were overt hypothyroidism and 15 with subclinical hypothyroidism. Among 40 males, 35 were having subclinical hypothyroidism and 5 overt hypothyroidism. So $60 \%$ had hypothyroidism among 100 cases. Among 60 cases of hypothyroidism in our study 40 were found to have coronary artery disease and increased chance of cardiovascular accidents. Patients with hypothyroidism was found to have increased risk of diabetes and hypertension. An increased prevalence of hypothyroidism was observed in patients with a more reduced glomerular filtration rate.

\begin{tabular}{|l|c|c|c|}
\hline Factors & $\begin{array}{c}\text { Normal TFT } \\
\text { values }\end{array}$ & $\begin{array}{c}\text { Subclinical } \\
\text { hypothyroidism }\end{array}$ & $\begin{array}{c}\text { Overt } \\
\text { hypothyroidism }\end{array}$ \\
\hline Gender & 18 & 37 & 5 \\
Male & 10 & 15 & 5 \\
Female & 20 & 29 & 6 \\
\hline Diabetes & 25 & 11 & 4 \\
Yes & & 32 & 13 \\
No & $\mathbf{1 5}$ & $\mathbf{6}$ & 2 \\
\hline Systemic hypertension & $\mathbf{1 2}$ & $\mathbf{2 8}$ & 12 \\
Yes & $\mathbf{7}$ & $\mathbf{1 5}$ & 5 \\
No & $\mathbf{3 3}$ & & \\
\hline Cardiovascular accidents & & \\
Yes & & \\
No &
\end{tabular}

Statistical Analysis: Analysis was performed with the SPSS statistical software package.
Descriptive statistics like mean, standard deviation and proportions were calculated. 


\section{Discussion}

Thyroid disorders and CKD are independently some of the most prominent medical conditions found in India The most common changes in CKD relating to the thyroid gland are of elevated TSH with low T3 levels (overt hypothyroidsim) and subclinical hypothyroidism. in our study also $60 \%$ had hypothyroidism. Majority of these patients belong to CKD4/5 stages. Prevalence of hypothyroidism increased with increasing severity of CKD. The TSH level is often elevated in CKD in response to thyrotropin from pituitary as a result of uremic effect. Chronic metabolic acidosis is also a contributing factor. in these patients, it was also observed to have increased cardiovascular accidents. There is also increased incidence of anemia among the hypothyroid patients compared to non hypothyroid CKD patients. Patients who receive appropriate treatment for their thyroid disease have a decreased chance of developing or exacerbating renal dysfunction.

\section{Conclusion}

The study concludes that there is signification association between hypothyroidism and end stage renal disease. There was also increased cardiovascular mortality in these patients. This study emphasis the need for routine thyroid hormone monitoring in CKD patients.

\section{References}

1. L. Luo, J. Z. Luo, and I. Jackson, "Tripeptide amide L-pyroglutamylhistidyl-L prolineamide (L-PHP Thyrotropin- Releasing Hormone, TRH) promotes insulin-producing cell proliferation," Current Aging Science, vol. 6, no. 1, pp. 8-13, 2013.

2. M. W. Szkudlinski, V. Fremont, C. Ronin, and B. D. Weintraub, "Thyroid-stimulating hormone and thyroid-stimulating hormone receptor structure-function relationships,"
Physiological Reviews, vol. 82, no. 2, pp. 473-502, 2002.

3. G. Basu and A. Mohapatra, "Interactions between thyroid disorders and kidney disease," Indian Journal of Endocrinology and Metabolism, vol. 16, no. 2, pp. 204213, 2012.

4. M. Chonchol, G. Lippi, G. Salvagno, G. Zoppini, M. Muggeo, and G. Targher, "Prevalence of subclinical hypothyroidism in patients with chronic kidney disease," Clinical Journal of the American Society of Nephrology, vol. 3, no. 5, pp. 12961300, 2008.

5. E. I. Feinstein, E. M. Kaptein, J. T. Nicoloff, and S. G. Massry, "Thyroid function in patients with nephrotic syndrome and normal renal function," American Journal of Nephrology, vol. 2, no. 2, pp. 70-76, 1982.

6. D. P. Sarma and G. T. Simmons, "Intraglomerular metastases from papillary carcinoma of the thyroid," The Journal of the Louisiana State Medical Society, vol. 141, no. 6, pp. 26-28, 1989.

7. A. Gamboa-Dominguez and A. TenorioVillalvazo, "Metastatic follicular variant of papillary thyroid carcinoma manifested as a primary renal neoplasm," Endocrine Pathology, vol. 10, no. 3, pp. 265-268, 1999.

8. Mohamed Mohamedali, Srikanth Reddy Maddika, Anix Vyas, Viswanathan Iyer, Pramil Cheriyath, "Thyroid Disorders and Chronic Kidney Disease", International Journal of Nephrology, vol. 2014, Article ID 520281, 6pages, 2014. https://doi.org/1 $0.1155 / 2014 / 520281$. 